\title{
An Unusual Cerebral Air Embolism Developing within the Posterior Circulation Territory after a Needle Lung Biopsy
}

\author{
Kentaro Suzuki, Masayuki Ueda, Kanako Muraga, Arata Abe, Satoshi Suda, \\ Seiji Okubo and Yasuo Katayama
}

\begin{abstract}
We herein report the case of a 75-year-old woman with a paradoxical cerebral air embolism (CAE). She developed a bilateral visual disturbance at the time of needle puncture during a computed tomography (CT)guided percutaneous needle lung biopsy in the face down position. The air density within the descending aorta on chest CT suggested the presence of a cerebral air embolism. Brain MRI demonstrated increased signal intensity in the bilateral occipital lobes on diffusion-weighted images. Usually, CAE occurs predominantly in the right hemisphere for anatomical reasons. The face down position and the anatomical features of the right subclavian artery, which diverges backward from the brachiocephalic artery, might explain such a unique distribution of CAE in this patient.
\end{abstract}

Key words: air embolism, cerebral infarction, needle biopsy, hyperbaric oxygen therapy

(Intern Med 52: 115-117, 2013)

(DOI: 10.2169/internalmedicine.52.8760)

\section{Introduction}

Cerebral air embolism (CAE) is a rare complication of trauma and surgery that results in cerebral infarction (1). Usually, CAE occurs predominantly in the right hemisphere due to the anatomical features of the branch of the aortic arch (2). We experienced a patient with CAE in the posterior circulation territory that developed after a computed tomography (CT)-guided percutaneous needle lung biopsy (CTNLB) who showed a dramatic response to hyperbaric oxygen (HBO) therapy. In this paper, the association between the unusual distribution of $\mathrm{CAE}$ and the posture at onset are discussed.

\section{Case Report}

A 75-year-old woman with a lung tumor underwent CTNLB in the face down position. She suddenly complained of dizziness during puncture and developed blindness thereafter. The initial neurological examination conducted 20 minutes after the onset of symptoms revealed a visual disturbance of the finger counting level. Electrocar- diogram showed a sinus rhythms, and there were no abnormalities in the coagulation system. Chest CT performed during the puncture displayed air density within the descending aorta (Figure A), although no air density was detected within the brain on initial CT performed 30 minutes after onset (Figure B). Initial brain magnetic resonance imaging (MRI) performed 60 minutes after onset demonstrated neither an abnormal flow signal on magnetic resonance angiography (MRA) (Figure C) nor abnormal lesions on diffusionweighted images (DWI) (Figure D). The presence of intraarterial air density on chest CT and the symptoms of dizziness with visual disturbance suggested a diagnosis of CAE, and the patient was treated with oxygen inhalation and intravenous injection of edaravone and heparin. Her visual impairment progressed to the light sense level by 12 hours after onset, and a second round of MRI was emergently performed, which demonstrated bilateral occipital high intensity on DWI (Figure E). Thereafter, HBO was administered according to the standard protocol (five hours at 1.9-2.8 atmosphere for five days). A third round of MRI performed five days after onset revealed the complete disappearance of the occipital abnormalities observed on the second MRI (Figure F). The occipital cortices showed no abnormalities 

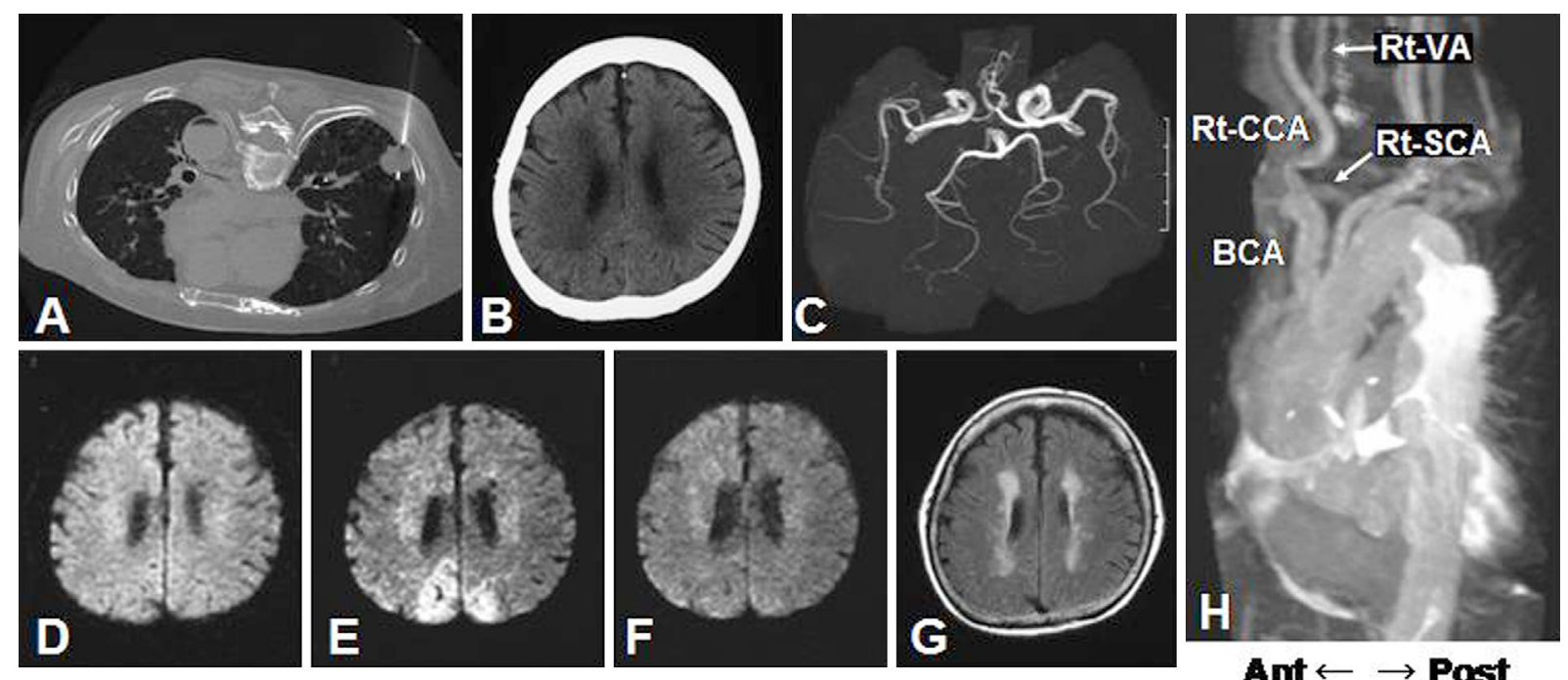

Figure. (A) Chest computed tomography (CT) image obtained during needle puncture displays air density within the descending aorta. (B) Initial brain CT performed 30 minutes after symptom onset shows white matter ischemic changes, but not any air density. (C) Initial magnetic resonance (MR) angiography shows normal flow signals in the intracranial large arteries. (D) Initial brain diffusion weighted MR image obtained 60 minutes after symptom onset demonstrates no acute ischemic changes. (E) Brain diffusion weighted MR image obtained 12 hours after symptom onset reveals high signal intensity lesions in the bilateral occipital lobes. (F) Brain diffusion weighted MR image obtained five days after symptom onset demonstrates that the occipital abnormalities observed on the second MRI were completely diminished. (G) Fluid attenuated inversion recovery image of the brain obtained five days after symptom onset demonstrates chronic ischemic changes in the white matter, but not in the occipital lobes. (H) Thoracic MR angiography shows that the right subclavian artery first diverged backward. Rt-BCA: right brachiocephalic artery, Rt-CCA: right common carotid artery, Rt-SCA: right subclavian artery, Rt-VA: right vertebral artery, Ant: anterior, Post: posterior

on fluid attenuated inversion recovery (FLAIR) images obtained during the series of MRI examinations (Figure G). The patient's visual symptoms improved after undergoing HBO and completely disappeared by day 7 .

\section{Discussion}

Systemic air embolisms are a rare complication of CTNLB, with an incidence of $0.02-0.07 \%$ (3-5). There are two causative mechanisms for the development of air embolisms after CTNLB: a direct communication between the atmosphere and the pulmonary vein may occur when the needle tip is placed in the pulmonary vein (6) and a bronchovenous fistula can be created when the needle passes through the lung parenchyma (7).

In one study, Griese et al. reported that 38 of 39 CAE patients showed predominant lesions in the right hemisphere after undergoing cardiosurgery (2). That the brachiocephalic artery is the first major branch of the aortic arch can explain the difference in prevalence of CAE between the hemispheres. In contrast, the present patient developed CAE within the bilateral occipital lobes. The involved territory might be associated with the face down position of the patient at onset. Intra-arterial air moves to the upper side of the vascular wall, easily flowing into the right vertebral and subclavian arteries in the face down position because the right subclavian artery diverges backward from the brachiocephalic artery. Thoracic MRA confirmed the presence of these anatomical features in the current patient (Figure $\mathrm{H}$ ).

$\mathrm{HBO}$ is recommended for the treatment of air embolisms (8-11). The high pressure reduces the volume of the air embolus, and the hyperoxygenation enables gas removal via denitrogenation, thereby maintaining oxygenation in the ischemic tissues. Although prompt initiation of $\mathrm{HBO}$ to treat CAE is preferred in order to prevent irreversible brain damage, the therapy is beneficial up to 30 hours (12). Indeed, HBO was initiated 13 hours after onset in the present case, and the patient's symptoms, as well as the DWI abnormalities, all completely disappeared.

The present case demonstrates that the face down position can result in the development of unusual occipital CAE lesions and indicates that a good response to $\mathrm{HBO}$ can be achieved even after treatment delay. Because CAE is a serious complication of CTNLB, clinicians should be therefore aware of the importance of this potential disorder.

The authors state that they have no Conflict of Interest (COI). 


\section{References}

1. Shaikh N, Ummunisa F. Acute management of vascular air embolism. J Emerg Trauma Shock 2: 180-185, 2009.

2. Griese H, Seifert D, Koerfer R. Cortical infarction following cardiosurgical procedures: air embolism as a probable cause. Eur Neurol 61: 343-349, 2009.

3. Tomiyama N, Yasuhara Y, Nakajima Y, et al. CT-guided needle biopsy of lung lesions: a survey of severe complication based on 9783 biopsies in Japan. Eur J Radiol 59: 60-64, 2006.

4. Richardson CM, Pointon KS, Manhire AR, Macfarlane JT. Percutaneous lung biopsies: a survey of UK practice based on 5444 biopsies. Br J Radiol 75: 731-735, 2002.

5. Sinner WN. Complications of percutaneous transthoracic needle aspiration biopsy. Acta Radiol Diagn (Stockh) 17: 813-828, 1976.

6. Westcott JL. Air embolism complicating percutaneous needle biopsy of the lung. Chest 63: 108-110, 1973.
7. Aberle DR, Gamsu G, Golden JA. Fatal systemic arterial air embolism following lung needle aspiration. Radiology 165: 351-353, 1987.

8. Bacha S, Annane D, Gajdos P. Iatrogenic air embolism. Presse Med 25: 1466-1472, 1996.

9. Dutka AJ. A review of the pathophysiology and potential application of experimental therapies for cerebral ischemia to the treatment of cerebral arterial gas embolism. Undersea Biomed Res 12: 403-421, 1985.

10. Kindwall EP. Massive surgical air embolism treated with brief recompression to six atmospheres followed by hyperbaric oxygen. Aerosp Med 44: 663-666, 1973.

11. McDermott JJ, Dutka AJ, Koller WA, Pearson RR, Flynn ET. Comparison of two recompression profiles in treating experimental cerebral air embolism. Undersea Biomed Res 19: 171-185, 1992.

12. Green BT, Tendler DA. Cerebral air embolism during upper endoscopy: case report and review. Gastrointest Endosc 61: 620-623, 2005.

(C) 2013 The Japanese Society of Internal Medicine http://www.naika.or.jp/imonline/index.html 